\title{
RNA-seq and two-dimensional gel electrophoresis revealed genomic and proteomic signatures during bacteriophage-host interactions in Pseudomonas aeruginosa
}

\section{Yinling Tan ( $\nabla$ tanyinling2019@163.com )}

Army Medical University

\section{Qiu Zhong}

Third Military Medical University Daping Hospital and Research Institute of Surgery

\section{Xia Zhao}

Army Medical University

\section{Canhuang Chen}

No. 180 Hospital of PLA

\section{Yang Li}

Third Military Medical University Daping Hospital and Research Institute of Surgery

Shuai Le

Army Medical University

\section{Ming Chen}

Third Military Medical University Southwest Hospital Department of Pathology

\section{Research article}

Keywords: Pseudomonas aeruginosa, bacteriophage, transcriptomics, two-dimensional electrophoresis

Posted Date: October 10th, 2019

DOI: https://doi.org/10.21203/rs.2.15950/v1

License: (c) (1) This work is licensed under a Creative Commons Attribution 4.0 International License. Read Full License 


\section{Abstract}

Background Understanding the biological nature of bacteriophage is important in exploring the therapeutic and biotechnological potentials of bacteriophages. However, available information is limited to the infection processes on either model phages infecting Escherichia coli or lytic phages against pathogens. The interplay between lysogenic phage and its host was rarely studied. Results We investigated the interactions between Pseudomonas aeruginosa and a lysogenic bacteriophage PaP3 through RNA-seq and two-dimensional gel electrophoresis (2D-GE). Compared to the uninfected host, a total of 2,891 (51.3\%) differentially expressed genes (DGEs) were identified, most of which were repressed by phages, including the changes in metabolic-related and virulence-associated genes. The RTqPCR results showed consistent directional changes compared with the RNA-seq results. According to 2D-GE, phage structure proteins were detected after phage infection. The host proteins, such as flagella hook-associated proteins, disappeared gradually after phage infection and may be shut off by phage. Conclusions All these indicate that although lysogenic phages do not immediately lyse the host, they play a significant regulatory role in the expression of host genes. Our findings provide an expanded view of the lysogenic phage infection processes and may offer potential targets for therapeutic intervention against P. aeruginosa infections.

\section{Background}

Pseudomonas aeruginosa, an opportunistic pathogen that often infects patients with cystic fibrosis or burns wounds, is becoming a public health concern due to its high rate of multidrug resistance [1]. In 2017, $P$. aeruginosa was listed as one of the 12 superbugs with the most severe drug resistance by the WTO[2]. $P$. aeruginosa phages genomics has been extensively studied due to their potential in treating $P$. aeruginosa infection [3]. And more than two hundred of $P$. aeruginosa phage genomes have been deposited in NCBI, includes dsDNA, dsRNA, and ssRNA phages [4]. besides, the therapeutic potential of $P$. aeruginosa phages has also been extensively explored in animal models [5, 6]. In 2018, the trial was stopped early due to some obstacles, but it is the hope of an anti-infection route [7]. Recently, the Engineered bacteriophages successfully treated a 15-year-old patient who was infected with mycobacterium[8]. However, a solid understanding of phage-host interactions at the molecular level is essential for future phage therapy applications. Moreover, these cellular processes that targeted by phages may be considered as potential antimicrobial drug targets[9]. Thus, the molecular interactions between phage and host is valuable for identifying potential antimicrobial target.

RNA-seq is a powerful technology to profile the genome-wide phage-host interactions during phage infection. Current RNA-seq analysis on phage-host interactions is largely based on Escherichia coli phages [10] or lytic phages infecting pathogens [11-17], however, limited data on the infection processes of lysogenic phages has been reported. P. aeruginosa lytic phage-host interactions have recently been revealed by different research groups. Chevallereau et al. (2016) found that the $P$. aeruginosa bacteriophage PAK_P3 can hijack RNA processing and significantly deplete bacterial transcripts to facilitate phage replication [12]. Zhao et al. (2017) also found that lytic phage PaP1 infection results in 
downregulation of 354 differentially expressed genes (DEGs)[18]. De Smet et al. (2016) revealed that metabolic impacts were highly phage-specific and that phage-encoded auxiliary metabolic genes can reprogram host metabolism in a phage-specific manner[11].

Our group has studied the interactions between the lysogenic phage PaP3 and its host through microarray [14]. We further elucidate this process through RNA sequencing (RNA-seq) and twodimensional gel electrophoresis (2D-GE). The transcriptomic data and 2D-GE results allowed us to gain a global view on the hindrances between phage and bacterial host interaction.

\section{Results}

\section{Analysis of the PA3 Gene Dynamic Expression after Phage Infection.}

According to the one-step growth curve of PaP3 and the dynamic infection cycle between phage and host, we examined the transcriptional changes at five time points after phage infection $(5,10,20,30$, and $80 \mathrm{~min}$ ) by using the RNA-seq analysis, and phage-uninfected host cells ( $0 \mathrm{~min}$ ) were used as controls. These time points contain the entire cycle of phage infection in the host and thus fully represent host gene expression. The six sequenced reads covered between $70 \%$ and $100 \%$ of genes, with an average of 4,298 genes, which accounted for $80 \%$ of all reference genes.

Relative to the uninfected host, a total of 2,962 DEGs were obtained according to the gene expression analysis; among these DEGs 2,891 and 71 were PA3 and PaP3 genes, respectively. The 2,891 DEGs (fold change $\geq 2, P<0.05$ ), included 1,550 up-regulated and 1368 down-regulated DEGs (Fig. 1). Moreover, most upregulated and downregulated genes are detected at 80 mins, when most cells are lysed and phages are released. Thus, the results from 80 mins might not be accurate.

Host phage regulation mainly occurred in the early logarithmic phase (10-20 min in this experiment). In the DEG analysis, 1,550 genes were upregulated, while 1,368 genes were downregulated. Analysis of the functions of these suppressed PA3 genes indicate that except for PA3 gene itself and a large proportion of undefined gene, the rest of the genes are mainly distributed in the transcriptional regulators (339/3863, $8.7 \%)$, amino acid synthesis and metabolism (185/3863, 4.7\%), post-translational modification and degradation (166/3863, 4.3\%), and energy metabolism (150/3863 3.8\%) (Fig. 2). These results indicate that PaP3 exerts a general inhibitory effect on host genome transcription.

A total of 71 phage genes were clustered by hierarchical cluster analysis based on the result of RNA-Seq analysis. It gathered into 3 clusters of genes, including 12 early genes (cluster1, at 5-10 min), 21 late genes (cluster 2, at 10-20 min) and 38 middle genes (cluster3, at 30-80 min) (Fig. 3). The results are consistent with microarray analysis results (Zhao et al., 2016b).

\section{RT-qPCR Validation of Selected DEGs.}

To validate DGEs identified by RNA-seq, we selected nine DEGs for RT-qPCR (Table 1), including hfq, katA, $\operatorname{dadA}$, flgK, groEL, flgL, lecA, rpoS, and rpoZ. Two Up-regulated genes involved stress reaction and Quorum 
sensing (like oxidation-reduction process $₫$ oxidation-reduction process $\$ through the entire infection process. While seven down-regulated included host biosynthetic process, Virulence factors, transcription and translation regulators, and metabolism-related genes囚occurs mainly in the middle and late stages of phage infection(30min, $80 \mathrm{~min}$ ). All RT-qPCR results show consistent directional changes compared with the RNA-seq results.

Table 1. RT-qPCR validation of the selected DEGs.

\begin{tabular}{ccccccccccc}
\hline \multirow{2}{*}{ Gene name } & \multicolumn{9}{c}{ Expression multiple } \\
& \multicolumn{1}{c}{ RNA-seq } & \multicolumn{7}{c}{ RT-qPCR } \\
\cline { 2 - 12 } & 5min & $10 \mathrm{~min}$ & $20 \mathrm{~min}$ & $30 \mathrm{~min}$ & $80 \mathrm{~min}$ & $5 \mathrm{~min}$ & $10 \mathrm{~min}$ & $20 \mathrm{~min}$ & $30 \mathrm{~min}$ & $80 \mathrm{~min}$ \\
\hline hfq & - & 0.322 & 0.204 & 0.332 & 0.105 & - & 0.448 & 0.222 & 0.018 & 0.020 \\
katA & - & 15.709 & 10.997 & - & - & - & 21.547 & 16.749 & - & - \\
\hline dadA & 0.203 & 0.141 & 0.108 & 0.159 & 0.148 & 0.368 & 0.251 & 0.039 & 0.039 & 0.041 \\
\hline flgK & - & 0.348 & 0.191 & 0.334 & 0.350 & - & 0.484 & 0.181 & 0.031 & 0.040 \\
\hline gro $E L$ & - & 0.400 & 0.414 & 0.493 & 0.295 & - & 0.442 & 0.304 & 0.062 & 0.034 \\
\hline flgL & - & 0.371 & 0.354 & 0.346 & 0.396 & - & 0.460 & 0.421 & 0.087 & 0.119 \\
\hline lecA $A$ & - & - & - & 9.781 & 5.708 & - & - & - & 2.231 & 2.102 \\
\hline ropS & - & - & 0.292 & 0.371 & 0.230 & - & - & 0.093 & 0.032 & 0.073 \\
\hline ropz & - & - & 0.369 & 0.038 & 0.089 & - & - & 0.294 & 0.021 & 0.022 \\
\hline
\end{tabular}

* 7 downregulated genes and 2 upregulated genes of $P$. aeruginosa were selected from the RNA-seq data set $(\mathrm{P}<0.05)$. The genes expression of DEGs are indicated as $\log _{2}$ (fold change) values.

\section{DEG KEGG Pathway Analysis in P. aeruginosa PA3 after Phage Infection.}

KEGG pathway significant enrichment analysis can identify the most important biochemical metabolic and signal transduction pathways (Table 2). The above DEGs that may be involved in the metabolic pathway were analyzed by combining the KEGG pathway database. As compared to the control group, a total of 2,962 DEGs were observed after phage PaP3 infected the host, and a total of 25 metabolic pathways were identified. The genes involved in differential expression are mainly involved in metabolic pathways, biosynthesis of secondary metabolites, and two-component systems. Pathways with enriched differential genes were mainly observed in the 30 - and 80 -min samples, further indicating that the

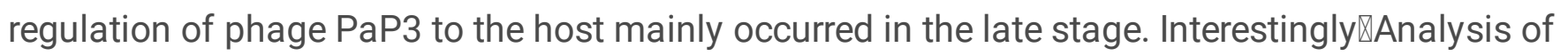
pathways enriched with differential gene expression in the late stage of phage infection show that the Cationic antimicrobial peptide (CAMP) resistance was significantly inhibited. Also, the suppressed the metabolism paths of essential amino acids (such as valine, leucine, isoleucine, tryptophan, lysine, histidine, arginine, and beta alanine), which were critical to bacterial life. In the aliphatic acid metabolic pathways, most of the relevant DEGs showed suppressed expression by inhibiting the gene expression of intermediates catalytic enzymes in aliphatic acid metabolism. This pathway involved two suppressing genes, namely, synthesizing aliphatic acid coenzyme FadD1 and FadD2. Mutations of these two enzymes not only lead to synthesis reduction of lipase, protease, rhamnolipid, and phospholipase in $P$. aeruginosa 
but also reduce the use of carbon sources in the environment [19]. The mentioned carbon sources include aliphatic acids and choline phospholipids (lung surface-active substance). Furthermore, bacterial movement and group movement are inhibited. FadD1 and FadD2 are also related to the virulence of $P$. aeruginosa, and the expression of these two enzymes are detected in cystic fibrosis cases $[20,21]$.

\section{Table 2. The KEGG pathway in P. aeruginosa PA3 after phage infection (percentage)}

\begin{tabular}{|c|c|c|c|c|c|}
\hline KEGG pathway & $5 \mathrm{~min}$ & $10 \mathrm{~min}$ & $20 \mathrm{~min}$ & $30 \mathrm{~min}$ & $80 \mathrm{~min}$ \\
\hline ABC transporters & & & $14.06 \%$ & $19.79 \%$ & $63.02 \%$ \\
\hline Alanine, aspartate and glutamate metabolism & & & & $32.43 \%$ & $75.68 \%$ \\
\hline Biosynthesis of antibiotics & & & & $19.33 \%$ & $58.40 \%$ \\
\hline Biosynthesis of secondary metabolites & & $5.64 \%$ & & $18.40 \%$ & \\
\hline Biosynthesis of unsaturated fatty acids & & & & $46.15 \%$ & \\
\hline Carbon metabolism & $10.16 \%$ & $7.81 \%$ & $17.19 \%$ & $25.00 \%$ & \\
\hline Cationic antimicrobial peptide (CAMP) resistance & & & $32.14 \%$ & $32.14 \%$ & $75.00 \%$ \\
\hline Degradation of aromatic compounds & & & & & $74.07 \%$ \\
\hline Fatty acid biosynthesis & & & & $42.31 \%$ & $76.92 \%$ \\
\hline Fatty acid degradation & $15.63 \%$ & & & & \\
\hline Fatty acid metabolism & $14.58 \%$ & & & $31.25 \%$ & \\
\hline Folate biosynthesis & & & & & $77.27 \%$ \\
\hline Glycolysis / Gluconeogenesis & & & & $32.43 \%$ & $70.27 \%$ \\
\hline Glyoxylate and dicarboxylate metabolism & & $10.53 \%$ & & & \\
\hline $\begin{array}{l}\text { Microbial metabolism in diverse environments } \\
\text { Monobactam biosynthesis }\end{array}$ & $8.37 \%$ & $6.08 \%$ & & $20.15 \%$ & $60.46 \%$ \\
\hline $\begin{array}{l}\text { Mionodactam blosynthesIS } \\
\text { Nitrogen metabolism }\end{array}$ & $18.92 \%$ & $18.92 \%$ & $27.03 \%$ & $29.73 \%$ & \\
\hline Oxidative phosphorylation & & & & & $69.81 \%$ \\
\hline Propanoate metabolism & & $12.82 \%$ & & & \\
\hline Pyruvate metabolism & & & & $26.67 \%$ & \\
\hline Ribosome & & & & & $67.65 \%$ \\
\hline Selenocompound metabolism & & & & $50.00 \%$ & \\
\hline Synthesis and degradation of ketone bodies & & $30.00 \%$ & & & \\
\hline Two-component system & & & $21.31 \%$ & $20.22 \%$ & \\
\hline Valine, leucine and isoleucine degradation & $14.58 \%$ & & & & \\
\hline
\end{tabular}

\section{Influence of Phage Infection on Host Virulence.}

The VFBD database revealed 251 virulence-related genes annotated in the PA01 strain genome and 115 genes differentially expressed after the bacteriophage PaP3 infected host PA3. Among these genes, 52 (45.2\%) were downregulated, including most flagella genes involved in adhesion (adherence), type IV pili biosynthesis and twitching motility-related genes and Hcp secretion island-1 encoded type VI secretion system (H-T6SS)[22]. Further analysis showed that the transcription factors controlling these virulence genes also presented low expression levels, suggesting that the phage PaP3 can globally manipulate the expression level of the host strain PA3 transcriptome through transcription factors with broad regulation. The upregulated genes in virulence-related genes included those involved in Phenazines biosynthesis with antimicrobial activity and alginate biosynthesis with antiphagocytosis, as well as genes involved in rhamnolipid and pyoverdine biosynthesis. The upregulated virulence-related genes also include the 
biotypes of $P$. aeruginosa type $\otimes$ secretion system from biosurfactants. These results suggest that the upregulated virulence genes may be involved in PA3 phage PaP3 immune resistance. In conclusion, phage infection may alter the virulence and resistance of hosts.

\section{Comparison of the Host DEGs from RNA-seq and Microarray Platforms.}

Globally, the numbers of DEGs in both microarray and RNA-seq analyses showed a considerable change in host transcription induced by phage. There were significant differences in the number of DEGs between microarray and RNA-seq. In RNA-seq there were 2,891 DEGs in the host (included 1,550 up-regulated genes and 1,368 down-regulated genes) खin the microarray analysis, there were 3,037 DEGs in the host (included 109 up-regulated genes and 2,928 down-regulated genes), a total of 1,186 DEGs were overlapped. The results from the two methods showed certain heterogeneity. However, the enrichment time of DEGs was consistent, which was the middle and late stage of infection. To further estimate the correlation of these genes called by both methods, we compared the estimated $\log _{2}$-fold changes of the consensus genes. The resulting correlations were mapped as scatter plots, and absolute levels of correlated gene expression were estimated in terms of the correlation coefficient $(r)$. The results showed Moderate positive correlation at each infection time point (Fig. 4). This comparison suggested that the absolute level of gene expression determined by both methods were correlated.

\section{Interaction of PA3 and Phage PaP3 at the Proteome Level.}

A total of 6 2D-GE maps were created using the samples from uninfected PaP3 cultures ( 0 min) and from infected PaP3 cultures at the time points of 5, 10, 20, 30, and $80 \mathrm{~min}$. These maps were individually matched with a reference gel map of uninfected PA3 cultures (Fig. 5A). Degradation of the three host protein spots was identified during phage infection; these three host proteins include FlgL (PA1087), FlgK (PA1086), and GroEL (PA4385). FlgL and FlgK are both flagellar hook-associated proteins that rapidly disappear from the 2D-GE map upon infection by PaP3. This result confirms the significant inhibition of mechanisms related to motility and attachment according to the RNA-seq data. GroEL is a heat-shock protein belonging to the HSP60 family, which can only be detected on the uninfected map as FlgL and FlgK[23]. Meanwhile, two PaP3 proteins, spot 1 (ORF06) and 2 (ORF16), were differentially expressed on infection maps. A highly abundant ORF06 (putative scaffold protein) appeared after 5 min of infection and continued to accumulate until 80 min of phage infection. ORF16 is a hypothetical protein that was detected only on the infection map, which disappeared from the other maps, at 5 min. Furthermore, high sequence identity $\left(E=3 \mathrm{e}^{-109}\right.$, identity $\left.=62 \%\right)$ was observed between ORF16 and the phage particle protein of Pseudomonas phage TL by NCBI BlastP analysis. Both ORF06 and ORF16 were classified as phage structure proteins. Thus, the structural proteins of phage PaP3 were detected in the early phase of infection. The expression level of these proteins was listed (Fig. 5B and 5C). The trend is overall fit between RNA-seq and the proteomic content, except phage protein Orf16, which disappeared in the gel but was upregulated in the RNA-seq data after 10 min.

\section{Discussion}


In this paper, we describe a $P$. aeruginosa lysogenic phage infection process through RNA-seq technology and 2D-GE-based whole-cell proteomics. In recent years, investigation on the transcriptional response of the host cell to phage infection is an area of extensive concern in transcriptome research by microarray or RNA-seq. A total of 105 references containing the words "transcriptome" and "bacteriophage" in the PubMed Central literature database are available to date, and most of the references describe host response to phage infection. In both the previous study and this work, a large number of PA3 phageinduced DEGs were found in the microarray and RNA-seq results. RNA-seq identified more significantly expressed DEGs, especially the upregulated genes as compared to the microarray. The results of both methods showed the same distributions of DEGs at each infection stage; The average DEG overlap was $38.4 \%$ and $53.9 \%$ for microarray and RNA-seq, respectively. The correlation of the fold-change ratios between the two technologies ( $r=0.53$ to 0.63 ) at different points is similar to other comparisons. The systematic differences in gene expression measurements can cause variations in the gene expression levels. Moreover, the different batches of RNA samples may partially explain the low overlap of DEGs from microarrays and RNA-seq. The microarray and RNA-seq data has been compared in other studies. The results had been reported as consistent [24]or moderately [25, 26]. Thus, further validations are needed before molecular investigations.

Phage genes can be classified into early ( $P$ 71-57), middle ( $P$ 56-22), and late phases ( $P$ 21-1) according to the expression pattern during infection as detected by RNA-seq. Phage proteins produced immediately after infection are likely to be involved in bacteriophage-host interactions. For example, phage proteins may protect the bacteriophage from bacterial defense mechanisms or may control the host metabolism to establish an efficient infection cycle. We identified an early phage-expressed protein 70.1, which directly binds to a global regulator RpoS to affect cell physiology, including impaired growth and reduced virulence[27]. Thus, a lot of phage early proteins appear to target different processes in the host cell. In addition, the early phage proteins exhibit antibacterial potentials.

The 2D-GE of cell lysates obtained before and after phage infection, followed by mass spectrometric identification allowed the detection of changes in the host protein expression caused by phage infection. The expression level of a host protein after infection indicates that the virus induces or inhibits its transcription, translation, or stability. Moderate changes were observed in 2D-GE gels. However, RNA-seq revealed a clear effect of phage PaP3 infection on host RNA expression. This result is likely due to a longer average half-life of bacterial proteins than the period of phage infection. Thus, downregulated host transcription not only results in a decrease in protein spots on the 2-DE gels after $80 \mathrm{~min}$. A total of two $\mathrm{PaP} 3$ proteins were significantly upregulated after phage infection. The PaP3 proteins are spot 1 (ORF06) and 2 (ORF16), which are annotated as a putative scaffold and hypothetical proteins, respectively. According to previous proteomic studies on phages, the most abundant proteins in the 2D-GE profiles are often phage structural proteins. A similar investigation was recently conducted using phage LUZ19- $P$. aeruginosa PAO1 model. A total of six induced host and five phage proteins (two structural proteins) were detected. Also, virtually no degradation of the host proteins was observed. In the present study, we also observed three significantly downregulated proteins, FlgL (PA1087), FlgK (PA1086), and GroEL (PA4385) (Fig. 5). These data revealed a significant inhibition on the motility and attachment of the host, as agreed 
with the RNA-seq data. However, the mechanism on how phage protein inhibits the motility of the host needs further investigation.

\section{Conclusions}

In this study, the interaction between PaP3 and host $P$. aeruginosa PA3 was analyzed by transcriptome sequencing which is verified at mRNA and protein levels (RT-PCR and two-dimensional protein electrophoresis). In addition, we compared and analyzed differences between microarray and RNA-seq at the transcriptome level, which provides new evidence for the verification of the two methods. The results showed that although PaP3 was a lysogenic phage, it had a great influence on the host. It could significantly down-regulate the genes of the host bacteria (especially in the middle and late stage), and plays an important role in the host metabolism and the expression of virulence genes. Our research not only enriches our biological understanding of lysogenic phages, but also may provide new targets for prevention and control of $P$. aeruginosa infections in the future.

\section{Materials And Methods}

\section{Bacterial Strains and Growth Conditions.}

The $P$. aeruginosa PA3 strain and the lysogenic phage PaP3 were maintained in our laboratory. $P$. aeruginosa strains were grown in Luria-Bertani (LB) medium (for broth culture) or 1.5\% (wt/vol.) agar LB plates at $37^{\circ} \mathrm{C}$. The $\mathrm{PaP} 3$ particles were collected and purified using $\mathrm{CsCl}$ gradient ultracentrifugation.

\section{One-Step Growth Curve.}

To determine the PaP3 one-step growth curve, we followed the methods described by Lu et al[28]. Briefly, PA3 logarithmic phase cultures (optical density at $600 \mathrm{~nm}$ [OD600] of 0.5) were infected with PaP3 (MOI [multiplicity of infection] of 10). After incubation at $37^{\circ} \mathrm{C}$ for $5 \mathrm{~min}$ to allow adsorption, the mixture was centrifuged for $30 \mathrm{~s}$ at $13000 \mathrm{~g}$. Unabsorbed phages were removed from supernatants by washing twice with LB medium. Sediments were suspended in $5 \mathrm{~mL} \mathrm{LB}$, and cultures were grown at $37^{\circ} \mathrm{C}$. The total time of adsorption and washing was approximately $10 \mathrm{~min}$. A total of $50 \mu \mathrm{L}$ of the sample was withdrawn every $10 \mathrm{~min}$, and the PaP3 particle count was determined using the double-layer agar plaque method. Burst time and size were calculated based on the one-step growth curve.

\section{RNA-seq and Quantitative Qeal-Time PCR (RT-qPCR).}

The samples for RNA-seq and RT-qPCR were prepared as previously described [14]. Briefly, a total volume of $10 \mathrm{~m}$ PA3 cultural $\left(\mathrm{OD}_{600}=0.5\right)$ was infected with phage PaP3 at MOI of 10 . For zboth RNA-seq analysis and RT-qPCR, the total RNA was isolated from six groups of bacterial cultures at $0,5,10,20,30$, and 60 min after phage infection by using an SV Total RNA Isolation System (Promega, USA, Z3100). RNA preparation and sequencing were conducted by BGI-Shenzhen using Illumina HiSeq 2000 platform (Shen Zhen, China). Reads were mapped to the P. aeruginosa PAO1 (NC_002516.2) and PaP3 
(NC_004466.2) reference genomes, respectively. Raw data from the RNA-seq analysis was deposited into the GEO database with the accession number GSE129181.In RT-qPCR analysis, cDNA synthesis was prepared using a PrimeScript RT reagent kit (Takara Bio, Dalian, China) according to manufacturer's recommendations. RT-qPCR was performed using the SYBR Premix Ex Taq II (Takara Bio). Primers used in this study are listed in Table 3. 16S rRNA was selected as the reference gene for normalization. The RTqPCR results were normalized using 16S rRNA and expressed as fold change (log2 scale) by the comparative $\mathrm{Ct}$ method. Control (0 $\mathrm{min})$ is normalized as 0 .

\section{Table 3. Primers used in this study}

\begin{tabular}{ll}
\hline Primers & Sequence $\left(5^{\prime}-\mathbf{3}^{\prime}\right)$ \\
\hline groEL-F & AGGGTGACAACGGGAGCAG \\
groEL-R & TCTCGATCATGTCGCCGTAC \\
\hline flgk-F & CTCAAGCCGCTGGGTATCA \\
\hline flgk-R & CGCCACCTTGTAGGTCTCGT \\
\hline dadA-F & GCGCCATCCCTTCATCG \\
\hline dadA-R & GATGTCGACGCGGTTGTTG \\
\hline hfa-F & AAAGGGCATTCGCTACAAGA \\
\hline hfq-R & AGATCGCGTGCTTGTAAACC \\
\hline katA-F & CAGCCATACCTTCAGCTTCATC \\
\hline katA-R & GATAGGTAGCCGCTTCCTTCTC \\
\hline rpoS-F & CATGTGGTCAAGGAGCTCAA \\
\hline rpoS-R & TATCCAGCAGGGTCTTGTCC \\
\hline rpoZ-F & GAAAGTGGCCTGGGAAAAC \\
\hline rpoZ-R & TACAGGGCCTCGGTGTTG \\
\hline lecA-F & GTGCGCTGGTCATGAAGAT \\
\hline lecA-R & CTGAACGAGCCGGAGTTATT \\
\hline flgL-F & GTGACCCTGGTGAATACCGC \\
\hline flgL-R & CGAATCTTGACGAAACTCTGCT \\
\hline 16s & AAGCAACGCGAAGAACCTTA \\
\hline 16s & CACCGGCAGTCTCCTTAGAG \\
\hline
\end{tabular}

\section{Data Analysis and Visualization.}

Sequencing reads were trimmed according to modified Phred quality scores of 0.5 by using CLC Genomics Workbench 6.51 (CLC Bio USA, Cambridge, MA). Data were normalized and analyzed using GeneSpring version GX10.0 (Agilent Technologies). DEGs exhibiting a twofold change in expression level at minimum were screened via one-way ANOVA $(P<0.05)$ with Benjamin-Hochberg correction for multiple comparisons[29]. Gene expression values were determined by the expected number of fragments per FPKM. R function cor was used for correlation analysis between RNA-seq and microarray and the dot plot were generated by ggplot2. Analysis of virulence-related genes was referred to the VFBD database (VFDB, http://www.mgc.ac.cn.cn/VFs/).

\section{D-GE for the Proteomic Analysis.}


The proteomic analysis was conducted by obtaining samples at 10-min intervals after PaP3 infection and performing two-dimensional electrophoresis (2D-GE) separations and image analyses on clear cell lysates[30]. 2D-GE gels were stained with silver, and images were acquired using the ImageScanner combined with LabScan software. The 2D-GE maps were analyzed, and spot data were generated using the standard spot detection parameters in the ImageMaster 2D platinum software. Differential spots were selected and identified using ESI-MS-MS[31].

\section{Declarations}

\section{Ethics approval and consent to participate}

Not applicable

\section{Availability of data and materials}

The reference genome and RNA-seq data used in this study can be found on NCBI. PaP3 and PA3 were isolated and stored in our laboratory, which are available from the corresponding author based on reasonable requests.

\section{Acknowledgments}

We thank professor Li Ming for his guidance in writing.

\section{Funding}

This work was supported by the Natural Science Foundation of China (NSFC, 31801037) and (NSFC, 31900145)

\section{Author information}

Department of Clinical Laboratory, Daping Hospital, Third Military Medical University (Army Medical university), Chongqing, 400042, China.

\section{Authors' Contributions}

The author(s) have made the following declarations about their contributions: Y.T., M.C., and Q.Z. Conceived and designed the experiments; Q.Z, X.Z, Y.L and C.C. performed the experiments; Q.Z and X.Z analyzed the data; S. L and Q.Z wrote the paper.

\section{Consent for publication}

Not applicable.

\section{Competing interests}


The authors declare that the research was conducted in the absence of any commercial or financial relationships that could be construed as a potential conflict of interest.a

\section{References}

1. Silby MW, Winstanley C, Godfrey SA, Levy SB, Jackson RW: Pseudomonas genomes: diverse and adaptable. FEMS Microbiol Rev 2011, 35(4):652-680.

2. Tacconelli E, Carrara E, Savoldi A, Harbarth S, Mendelson M, Monnet DL, Pulcini C, Kahlmeter G, Kluytmans J, Carmeli Y et al: Discovery, research, and development of new antibiotics: the WHO priority list of antibiotic-resistant bacteria and tuberculosis. Lancet Infect Dis 2018, 18(3):318-327.

3. Debarbieux L, Leduc D, Maura D, Morello E, Criscuolo A, Grossi O, Balloy V, Touqui L: Bacteriophages can treat and prevent Pseudomonas aeruginosa lung infections. J Infect Dis 2010, 201(7):10961104.

4. De Smet J, Hendrix H, Blasdel BG, Danis-Wlodarczyk K, Lavigne R: Pseudomonas predators: understanding and exploiting phage-host interactions. Nat Rev Microbiol 2017, 15(9):517-530.

5. Can K, Aksu U, Yenen OS: Investigation of PhiKZ phage therapy against Pseudomonas aeruginosa in mouse pneumonia model. Turk J Med Sci 2018, 48(3):670-678.

6. Cafora M, Deflorian G, Forti F, Ferrari L, Binelli G, Briani F, Ghisotti D, Pistocchi A: Phage therapy against Pseudomonas aeruginosa infections in a cystic fibrosis zebrafish model. Sci Rep 2019, 9(1):1527.

7. Jault P, Leclerc T, Jennes S, Pirnay JP, Que YA, Resch G, Rousseau AF, Ravat F, Carsin H, Le Floch R et al: Efficacy and tolerability of a cocktail of bacteriophages to treat burn wounds infected by Pseudomonas aeruginosa (PhagoBurn): a randomised, controlled, double-blind phase 1/2 trial. Lancet Infect Dis 2019, 19(1):35-45.

8. Hodyra-Stefaniak K, Lahutta K, Majewska J, Kazmierczak Z, Lecion D, Harhala M, Keska W, Owczarek $B$, Jonczyk-Matysiak E, Klopot A et al: Bacteriophages engineered to display foreign peptides may become short-circulating phages. Microb Biotechnol 2019, 12(4):730-741.

9. Liu J, Dehbi M, Moeck G, Arhin F, Bauda P, Bergeron D, Callejo M, Ferretti V, Ha N, Kwan T et al: Antimicrobial drug discovery through bacteriophage genomics. Nat Biotechnol 2004, 22(2):185-191.

10. Baxter K, Lee J, Minakhin L, Severinov K, Hinton DM: Mutational analysis of sigma70 region 4 needed for appropriation by the bacteriophage T4 transcription factors AsiA and MotA. J Mol Biol 2006, 363(5):931-944.

11. De Smet J, Zimmermann M, Kogadeeva M, Ceyssens PJ, Vermaelen W, Blasdel B, Bin Jang H, Sauer $\mathrm{U}$, Lavigne R: High coverage metabolomics analysis reveals phage-specific alterations to Pseudomonas aeruginosa physiology during infection. ISME J 2016, 10(8):1823-1835.

12. Chevallereau A, Blasdel BG, De Smet J, Monot M, Zimmermann M, Kogadeeva M, Sauer U, Jorth $P$, Whiteley M, Debarbieux L et al: Next-Generation "-omics" Approaches Reveal a Massive Alteration of 
Host RNA Metabolism during Bacteriophage Infection of Pseudomonas aeruginosa. PLoS Genet 2016, 12(7):e1006134.

13. Leskinen K, Blasdel BG, Lavigne R, Skurnik M: RNA-Sequencing Reveals the Progression of PhageHost Interactions between phiR1-37 and Yersinia enterocolitica. Viruses 2016, 8(4):111.

14. Zhao X, Chen C, Shen W, Huang G, Le S, Lu S, Li M, Zhao Y, Wang J, Rao X et al: Global Transcriptomic Analysis of Interactions between Pseudomonas aeruginosa and Bacteriophage PaP3. Sci Rep 2016, 6:19237.

15. Lavigne R, Lecoutere E, Wagemans J, Cenens W, Aertsen A, Schoofs L, Landuyt B, Paeshuyse J, Scheer $\mathrm{M}$, Schobert $\mathrm{M}$ et al: A multifaceted study of Pseudomonas aeruginosa shutdown by virulent podovirus LUZ19. MBio 2013, 4(2):e00061-00013.

16. Ravantti JJ, Ruokoranta TM, Alapuranen AM, Bamford DH: Global transcriptional responses of Pseudomonas aeruginosa to phage PRR1 infection. J Virol 2008, 82(5):2324-2329.

17. Blasdel BG, Chevallereau A, Monot M, Lavigne R, Debarbieux L: Comparative transcriptomics analyses reveal the conservation of an ancestral infectious strategy in two bacteriophage genera. ISME J 2017, 11(9):1988-1996.

18. Zhao X, Shen M, Jiang X, Shen W, Zhong Q, Yang Y, Tan Y, Agnello M, He X, Hu F et al: Transcriptomic and Metabolomics Profiling of Phage-Host Interactions between Phage PaP1 and Pseudomonas aeruginosa. Front Microbiol 2017, 8:548.

19. Feng S, Xu C, Yang K, Wang H, Fan H, Liao M: Either fadD1 or fadD2, Which Encode acyl-CoA Synthetase, Is Essential for the Survival of Haemophilus parasuis SC096. Front Cell Infect Microbiol 2017, 7:72.

20. Kang Y, Zarzycki-Siek J, Walton CB, Norris MH, Hoang TT: Multiple FadD acyl-CoA synthetases contribute to differential fatty acid degradation and virulence in Pseudomonas aeruginosa. PLOS One 2010, 5(10):e13557.

21. Yuan Y, Leeds JA, Meredith TC: Pseudomonas aeruginosa directly shunts beta-oxidation degradation intermediates into de novo fatty acid biosynthesis. J Bacterio/ 2012, 194(19):5185-5196.

22. Chen L, Yang J, Yu J, Yao Z, Sun L, Shen Y, Jin Q: VFDB: a reference database for bacterial virulence factors. Nucleic Acids Res 2005, 33(Database issue):D325-328.

23. Sipos A, Klocke M, Frosch M: Cloning and sequencing of the genes coding for the 10- and 60-kDa heat shock proteins from Pseudomonas aeruginosa and mapping of a species-specific epitope. Infect Immun 1991, 59(9):3219-3226.

24. Grabowiecka E, Martin D, Crozier L, Holden N: Escherichia coli 0157:H7 transcriptome datasets for comparison of RNA-seq and microarray platforms. Data Brief 2019, 22:126-131.

25. Rao MS, Van Vleet TR, Ciurlionis R, Buck WR, Mittelstadt SW, Blomme EAG, Liguori MJ: Comparison of RNA-Seq and Microarray Gene Expression Platforms for the Toxicogenomic Evaluation of Liver From Short-Term Rat Toxicity Studies. Front Genet 2018, 9:636.

26. Wolff A, Bayerlova M, Gaedcke J, Kube D, Beissbarth T: A comparative study of RNA-Seq and microarray data analysis on the two examples of rectal-cancer patients and Burkitt Lymphoma cells. 
PLoS One 2018, 13(5):e0197162.

27. Zhao X, Chen C, Jiang X, Shen W, Huang G, Le S, Lu S, Zou L, Ni Q, Li M et al: Transcriptomic and Metabolomic Analysis Revealed Multifaceted Effects of Phage Protein Gp70.1 on Pseudomonas aeruginosa. Front Microbiol 2016, 7:1519.

28. Lu S, Le S, Tan Y, Zhu J, Li M, Rao X, Zou L, Li S, Wang J, Jin X et al: Genomic and proteomic analyses of the terminally redundant genome of the Pseudomonas aeruginosa phage PaP1: establishment of genus PaP1-like phages. PLoS One 2013, 8(5):e62933.

29. Hochberg Y, Benjamini Y: More powerful procedures for multiple significance testing. Stat Med 1990, 9(7):811-818.

30. Velapatino B, Zlosnik JE, Hird TJ, Speert DP: Total protein extraction and 2-D gel electrophoresis methods for Burkholderia species. J Vis Exp 2013(80):e50730.

31. Xiao H, Zhang L, Zhou H, Lee JM, Garon EB, Wong DT: Proteomic analysis of human saliva from lung cancer patients using two-dimensional difference gel electrophoresis and mass spectrometry. Mol Cell Proteomics 2012, 11(2):M111 012112.

\section{Figures}


A

Time $\square 05 \mathrm{~min} \square 10 \mathrm{~min} \square 20 \mathrm{~min} \square 30 \mathrm{~min} \quad 80 \mathrm{~min}$

Hypothetical, unclassified, unknown

Membrane proteins

Putative enzymes

Transport of small molecules

Transcriptional regulators

Carbon compound catabolism

Energy metabolism

Adaptation, Protection

Cell wall / LPS / capsule

Amino acid biosynthesis and metabolism

Central intermediary metabolism

Two-component regulatory systems

Biosynthesis of cofactors, prosthetic groups and carriers

Translation, post-translational modification, degradation

Fatty acid and phospholipid metabolism

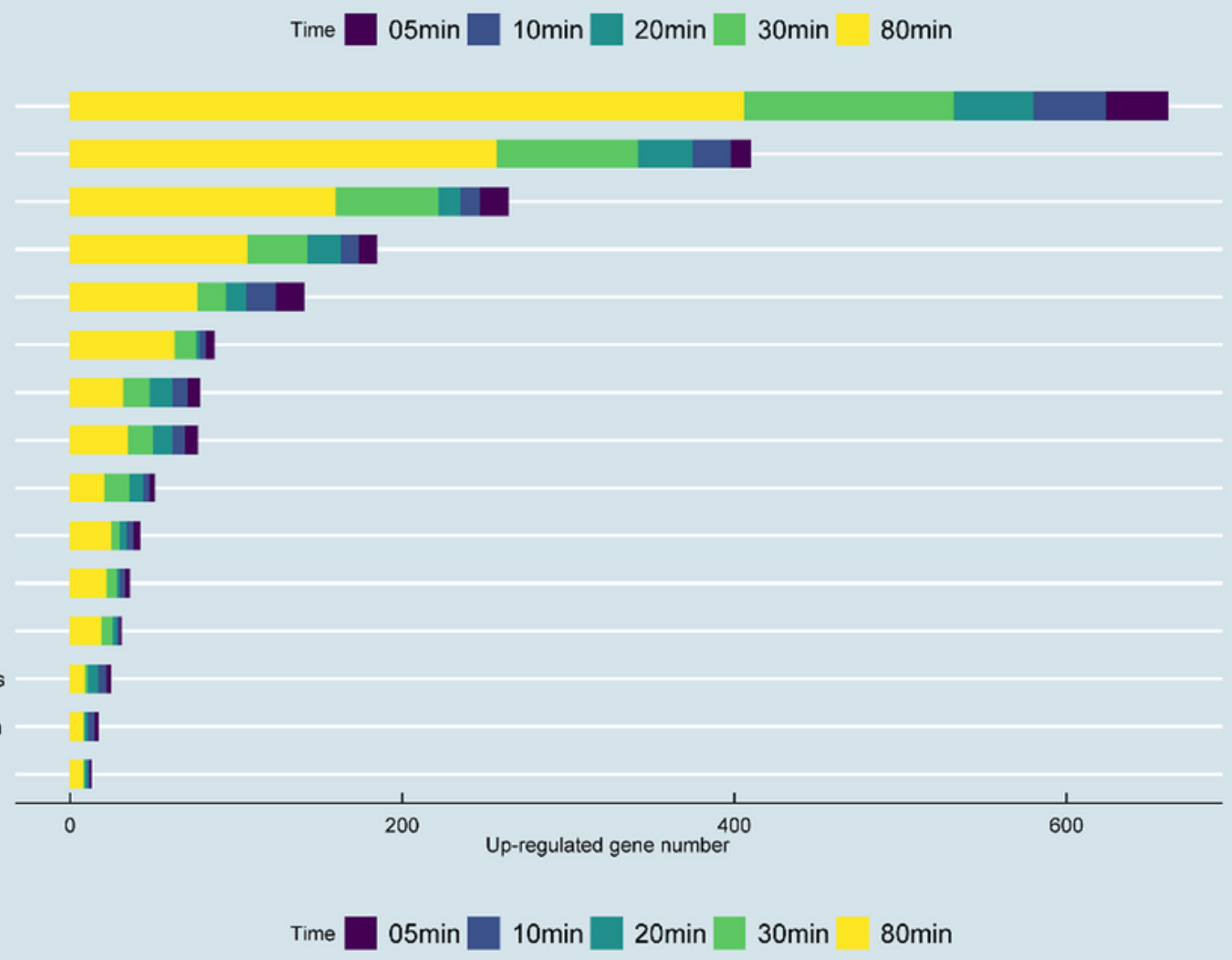

B

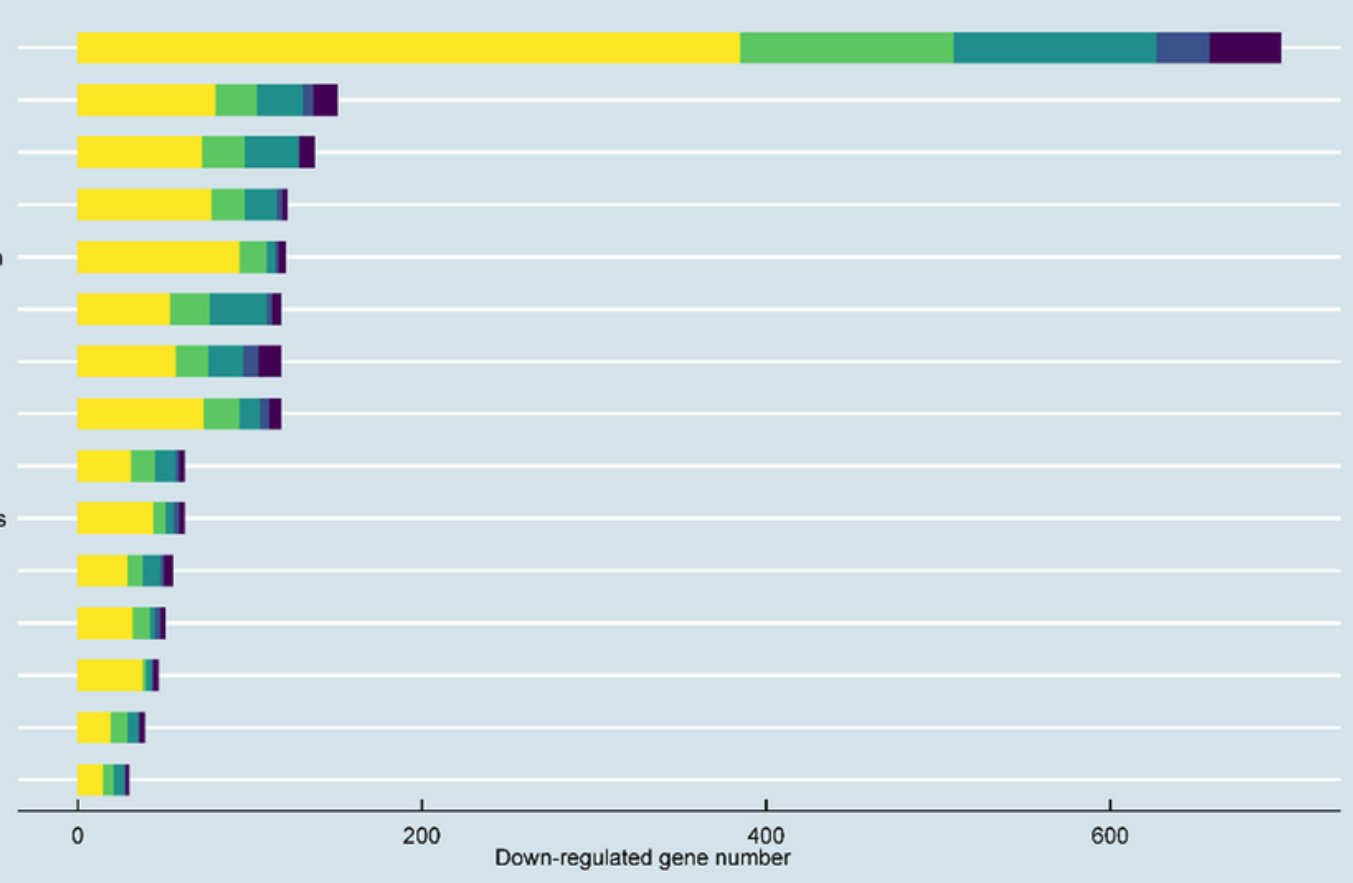

Hypothetical, unclassified, unknown

Membrane proteins

Transcriptional regulators

Amino acid biosynthesis and metabolism

Translation, post-translational modification, degradation

Transport of small molecules

Putative enzymes

Energy metabolism

Central intermediary metabolism

Biosynthesis of cofactors, prosthetic groups and carriers

Adaptation, Protection

Nucleotide biosynthesis and metabolism

Cell wall / LPS / capsule

Fatty acid and phospholipid metabolism

Carbon compound catabolism

Down-regulated gene number

\section{Figure 1}

Dynamic changes induced by PaP3 on the host gene expression. The dotted line represents a one-step growth curve of phage PaP3 in the host $\mathrm{P}$. aeruginosa PA3. The histogram represents the number of DEGs in PA3 at different time points after phage PaP3 infection compared with uninfected PA3. 


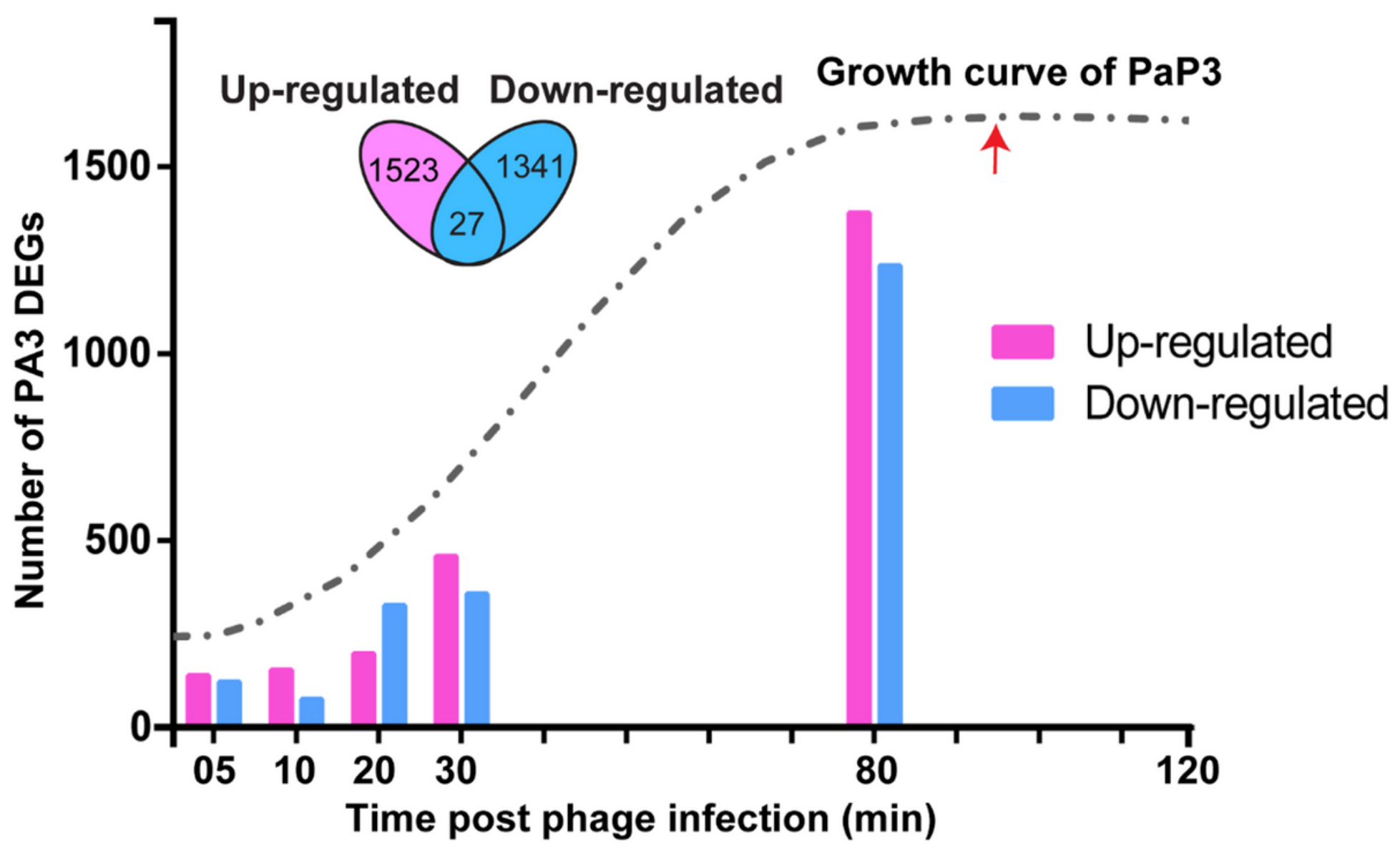

Figure 2

Classification of DEGs in P. aeruginosa PA3 after phage infection. Up-regulated (A) and down-regulated (B) genes at each time point are classified into 20 PseudoCAP annotations. 
A

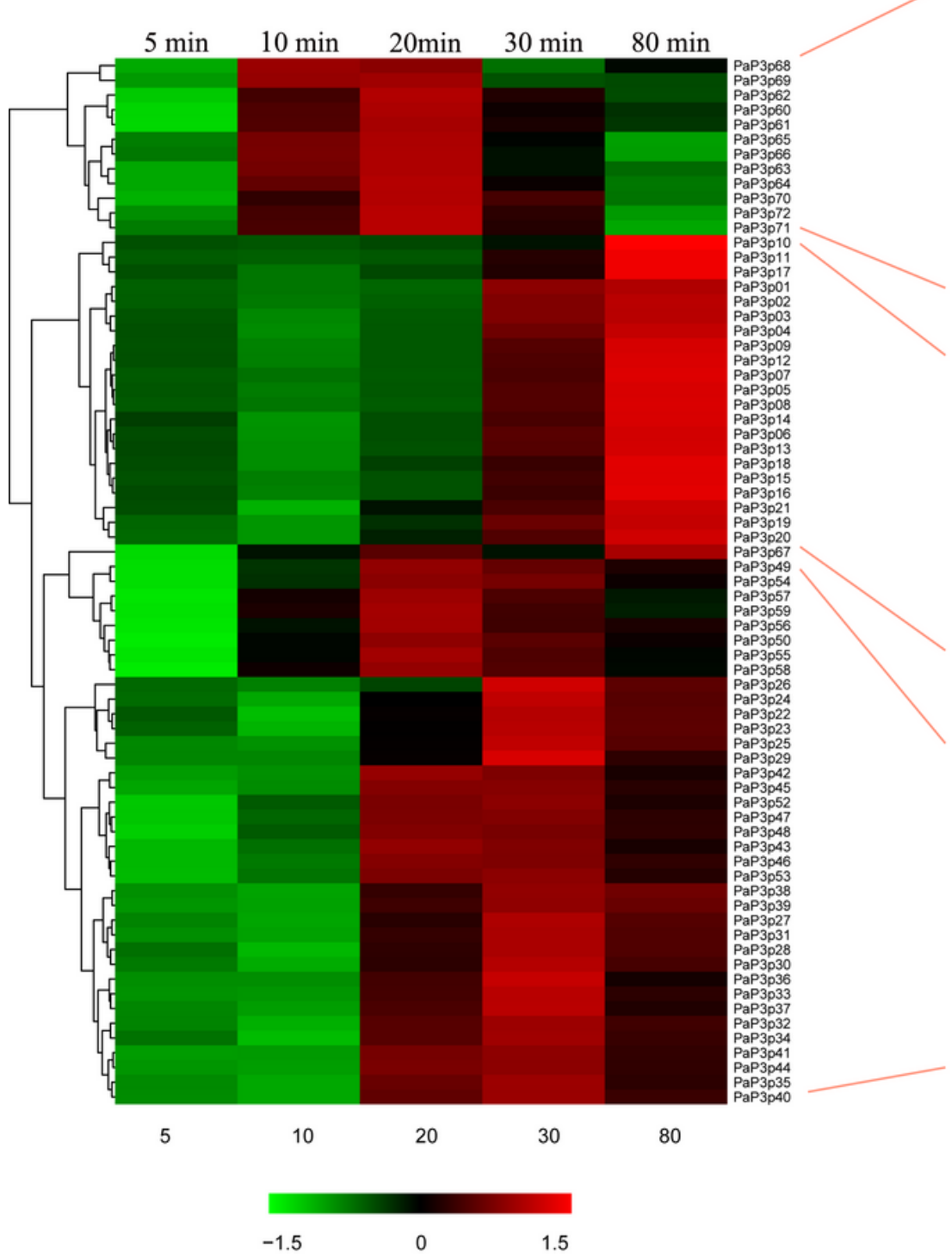

B
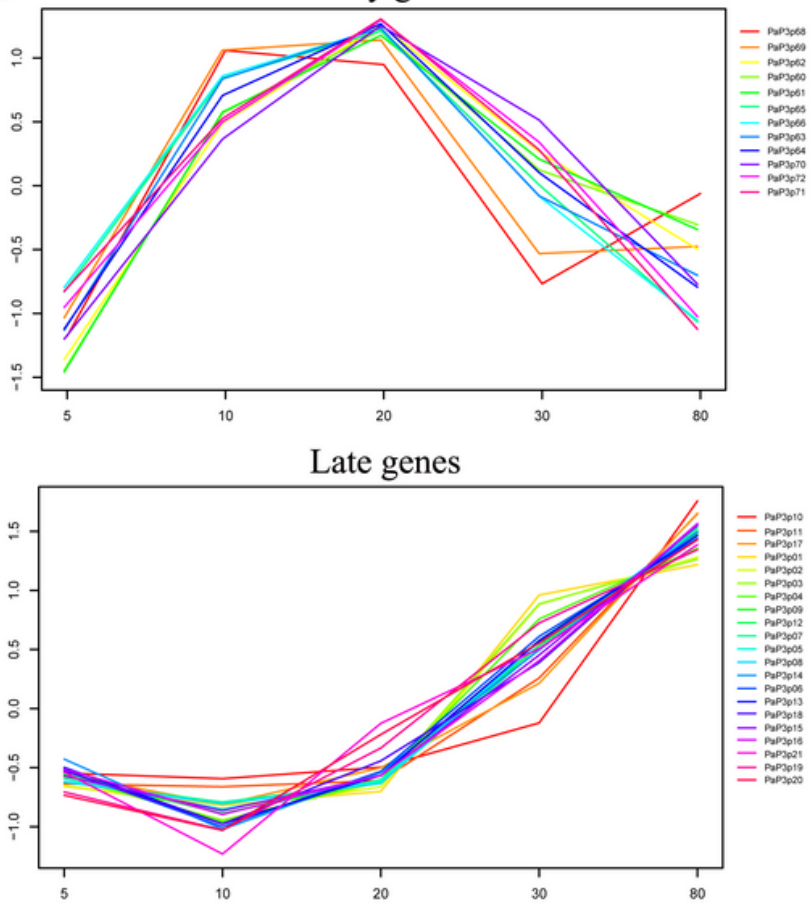

Middle genes

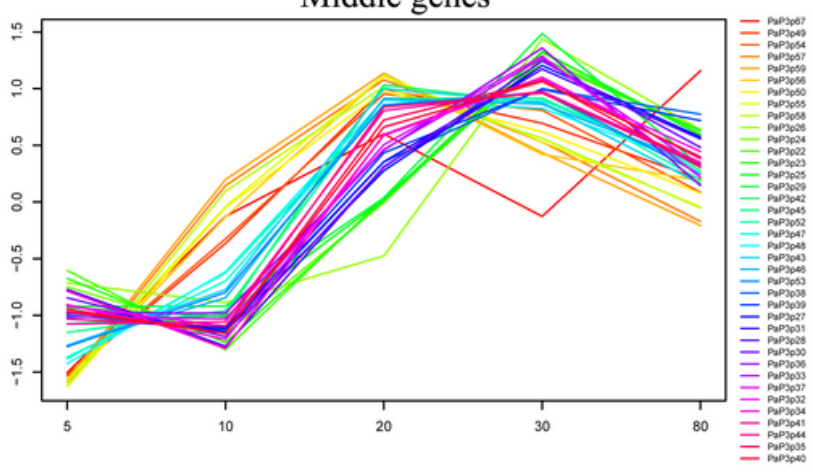

\section{Figure 3}

Transcriptomic profile of PaP3 genes. (A)Hierarchical cluster heat map of PaP3 genes. (B) The expression level of each cluster of the PaP3. The hierarchical cluster analysis was performed using ward.D2 and Minkowski methods based on the FPKM values of genes. A total of 71 PaP3 genes were clustered into 3 clusters. 


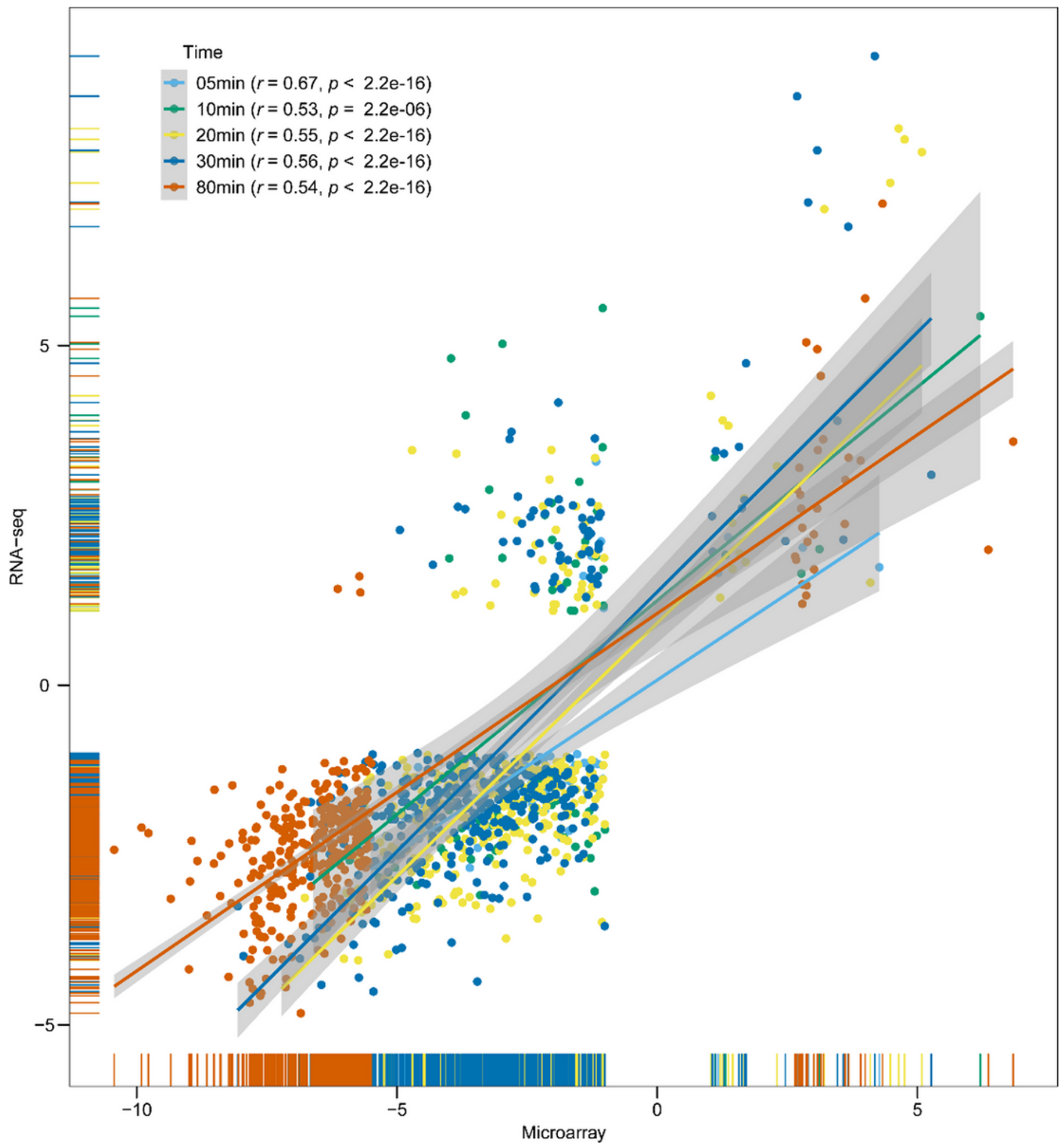

Figure 4

Comparative analysis of the microarray and RNA-seq methods. Scatter plot showing the relative expression levels of DEGs as log2-fold changes. Correlation between the two platforms is shown by the correlation coefficient (r). 
A
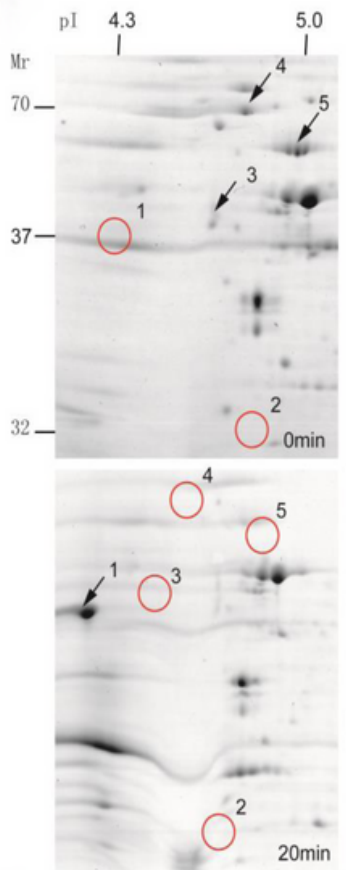

$\mathrm{C}$

\begin{tabular}{|c|c|c|c|c|c|c|}
\hline \multirow{2}{*}{$\begin{array}{l}\text { Spot } \\
\text { No. }\end{array}$} & \multirow{2}{*}{$\begin{array}{c}\text { MS/MS } \\
\text { Search score }\end{array}$} & \multirow{2}{*}{$\begin{array}{l}\text { AA } \% \\
\text { coverage }\end{array}$} & \multicolumn{2}{|c|}{ Protein size } & \multirow[t]{2}{*}{ Gene and product } & \multirow[t]{2}{*}{ PseudoCAP Function Class } \\
\hline & & & MW (Da) & PI & & \\
\hline 1 & 151.99 & 26 & 37599.4 & 4.32 & orf6, putative scaffold protein & \\
\hline 2 & 41.24 & 8 & 32094.4 & 4.8 & orf 16 , hypothetical protein & \\
\hline 3 & 76.2 & 12 & 46844.9 & 4.5 & $\begin{array}{l}\text { flgL, flagellar hook-associated } \\
\text { protein type } 3 \mathrm{FlgL}\end{array}$ & $\begin{array}{c}\text { Cell wall/LPS/capsule Motility \& } \\
\text { Attachment }\end{array}$ \\
\hline 5 & 249.8826 & 33 & 57086.2 & 5.04 & groEL, GroEL protein & Chaperones \& heat shock proteins \\
\hline
\end{tabular}

B
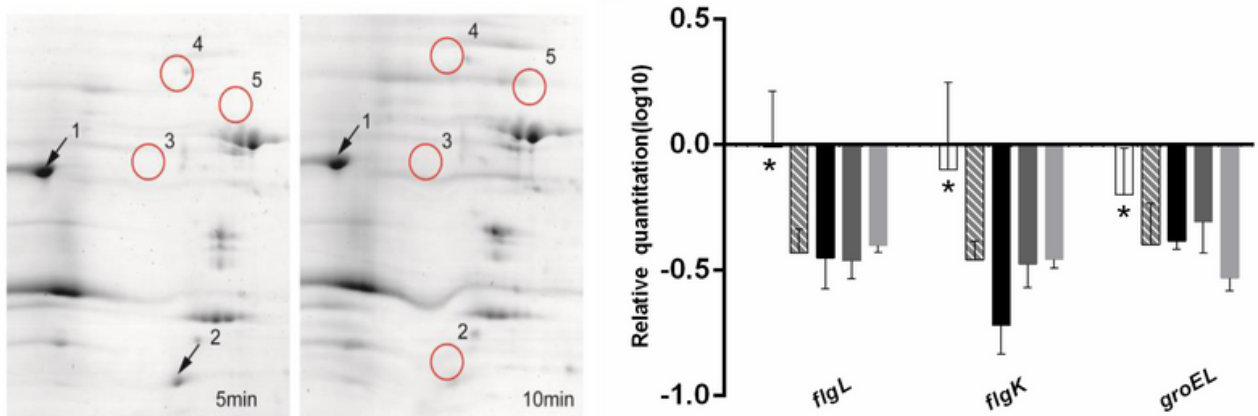

$5 \mathrm{~min}$

AIV $10 \mathrm{~min}$

- $20 \mathrm{~min}$

$30 \mathrm{~min}$

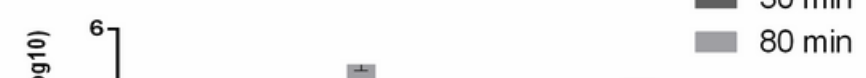

$00 \mathrm{~min}$

\section{Figure 5}

2D-GE maps validation of the selected DEGs at distinct time points. (A) Comparative analysis of differentially expressed proteins from uninfected PA3 ( $0 \mathrm{~min}$ ) and infected PA3 cells ( $5 \mathrm{~min}$ to $80 \mathrm{~min}$ ). Differentially expressed proteins that appeared on the map are indicated by red cycles, whereas the corresponding proteins that disappeared are indicated by black arrows. (B) The relative quantitation of the RNA-Seq results were expressed as fold change (log10 scale) by the comparative Ct method. Control ( $0 \mathrm{~min}$ ) is normalized as 0 . (C) A list of the protein spots was changed. 